\title{
Proposal of a P300-based BCI speller using a predictive text system
}

\author{
Ricardo Ron-Angevin and Leandro da Silva-Sauer \\ Departamento de Tecnología Electrónica, E.T.S.I. Telecomunicación \\ University of Málaga, Campus de Teatinos s/n, 29071 Málaga, Spain. \\ \{rron,sauer\}@uma.es
}

\begin{abstract}
Keywords: Brain-computer interface (BCI), P300, Speller, T9 interface
Abstract: $\quad$ This paper presents a P300-based BCI speller system that uses a virtual $4 \times 3$ keyboard based on the T9 interface developed on mobile phones in order to increase the writing speed. To validate the effectiveness of the proposed BCI, we compared it with two adaptations of the classical Farwell and Donchin speller, which is based on a 6 x 6 symbol matrix. Three healthy subjects took part in the experiment. The preliminary results confirm the effectiveness of T9-based speller, since the time needed to spell words and complete sentences was considerably reduced.
\end{abstract}

\section{INTRODUCTION}

People suffering from neurodegenerative disorders, such as amyotrophic lateral sclerosis, can eventually present great disabilities, particularly those involving the motor system. In some cases, such deficiencies can be really severe, to the point of causing total loss of control of the muscles that are responsible of voluntary body movements, including eye movement and breathing itself. People suffering from such disabilities lose all possibility to communicate with the external world via common muscular channels. Their only alternative is to use a brain-computer interface (BCI) system (Wolpaw et al., 2002; Birbaumer, 2006), which transforms brain activity into commands that are interpreted by a machine. Such a system offers a non-muscular channel for these users to interact with their environment, thus providing them with greater autonomy in their daily lives.

The most widely used BCI systems are those based on electroencephalographic (EEG) signal recording, due to its non-invasiveness, but also to its good temporal resolution and ease of use. Three types of EEG-based BCI systems have been used for communication purposes, namely those based on: (a) slow cortical potentials (SCPs), (b) P300 event-related potentials (ERP), and (c) sensorimotor rhythms (SMR) (Mak and Wolpaw, 2009). BCIs based on SCP and SMR demand that users are extensively trained before they show sufficient control of their brain activity. In contrast, BCIs based on P300 rely on a common, expected human response to infrequent tar- get stimuli-usually visual-and thus require minimal training. The P300 signal, recorded over the central and parietal regions, is a positive deflection of brain wave at a latency of about $300 \mathrm{~ms}$ after stimulus presentation.

The main applications of P300-based BCI systems are aimed at communication purposes. They are based on the P300 speller first developed by Donchin et al. (2000), which is still referenced and intensely studied (Bianchi et al., 2010; Kleih et al., 2010; Krusienski et al., 2008; Sellers et al., 2006). In this BCI, a $6 \times 6$ matrix of letters, arranged in rows and columns, is shown to the subject. The user focuses his/her attention on the matrix element he/she wishes to select as each row and column is flashed (i.e., intensified) randomly, one after the other. After a number of flashes, the symbol that the user was supposedly attending at is presented on screen.

The efectiveness of the P300-based BCI speller system is guaranteed by a number of studies carried out not only on healthy subjects (Donchin et al., 2000; Wang et al., 2005) but also on subjects affected by some motor disability (Sellers and Donchin, 2006). Overall, these studies conclude that the P300 speller processor is an effective communication tool for people who have lost or are losing their ability to write or speak. However, it is still needed to improve the usability of these BCI speller systems. Some factors, such as the mental fatigue induced by a long use (Kececi et al., 2006; Murata and Uetake, 2001), the sustained attention at a symbol on screen (Mangun and Buck, 1998), the user motivation (Kececi et al., 2006; 
Kleih et al., 2010) or his/her frustration due to a mistake (Kleih et al., in press) can influence the amplitude and latency of the P300 component (See Polich and Kok, 1995, for a review). In this regard, the influence on performance of the temporal and spatial aspects of the user interfaces of these systems is increasingly drawing the attention of researchers (Lu et al., 2013; McFarland et al., 2011; Shih et al., in press)

This paper presents a study aimed at contributing to this line of research. We propose a P300-based BCI speller system that uses the T9 interface developed for mobile phones. The virtual keyboard of this interface consisted of a $4 \times 3$ matrix, which was based on the one originally developed for mobile phones. Compared to the $6 \times 6$ matrix used by conventional P300based spellers (Sellers et al., 2006), using a smaller matrix was expected not only to lead to a higher accuracy, but also to reduce the time required to select a character, since the number of rows and columns that had to be flashed to detect it was reduced. On the other hand, we provided this speller with a text predictive system because selecting some characters in a 4 $x 3$ matrix would demand more than one choice from the user. By using such a system, those extra choices would hardly be necessary, thus increasing the user's writing speed (as discussed by Dunlop and Crossan (2000)). To validate the effectiveness of the proposed BCI, we compared it with two adaptations of the classical Farwell and Donchin speller in terms of the time needed to write a target sentence.

\section{METHOD}

\subsection{Participants, procedure and data acquisition}

Three healthy subjects (one man, aged 36, and two women, aged 22 and 25) took part in the experiment. None of them had previous experience with BCI systems. The experiment was divided into three sessions. The objective of each session was to evaluate one of the proposed interfaces, namely the two adaptations of the classical speller (i.e., the Spellermod and the SpellermodPred) and a speller based on the T9 interface (i.e., the SpellerT9). A description of these three interfaces will be done in the following sections.

Each participant carried out the three sessions in different days. To avoid effects due to learning, the order in which participants completed the three sessions was randomized.

Each session was divided into two phases: a first one for calibration purposes and a second one to eval- uate the interface. Participants were instructed to silently count how many times the selected symbol was intensified (i.e., flashed). In the calibration phase, they were asked to copy five sequences of three to four characters. In the evaluation phase, they were asked to write the sentence "Experiencia BCI en la Universidad de Málaga" (i.e., BCI experience at the University of Málaga).

As we wanted to compare the three interfaces in terms of the writing speed their users could achieve, all the temporary parameters related to the selection of a symbol (i.e., a letter) were equal across them. Specifically, in order to select a character, each row and each column was flashed 20 times, in a random order. The interstimulus interval (ISI) between flashes was $125 \mathrm{~ms}$. Once a symbol had been selected, a new sequence of flashes started after a pause of $2 \mathrm{~s}$. It is important to notice that the duration of a sequence of flashes depended on the size matrix, so that bigger matrices entailed longer sequences.

Eight-channel EEG data were recorded at the electrodes $\mathrm{Fz}, \mathrm{Cz}, \mathrm{Pz}, \mathrm{Oz}, \mathrm{P} 3, \mathrm{P} 4, \mathrm{PO} 7$ and PO8according to the $10 / 20$ international system-, with $\mathrm{FPz}$ as reference and the left mastoid as ground. Data were acquired by a biosignal amplifier (g.BSamp, Guger Technologies) with a sampling rate of $256 \mathrm{~Hz}$ and a 12-bit resolution data acquisition NI-USB-6210 (National Instruments) card.

\subsection{System description}

The three P300-based spellers were implemented through the BCI2000 platform (Schalk et al., 2004). The first speller, called Spellermod, was an adaptation of the classical Farwell and Donchin speller. Unlike this, which consisted of a 6 × 6 symbol matrix, the Spellermod presented a 7 x 6 virtual keyboard, in which additional symbols were included. We will refer to the Spellermod as the reference speller. The second speller, called SpellermodPred, was identical to the previous one save for the fact that it included a text predictive system in order to reduce the time needed to write. Finally, the third speller, called SpellerT9, consisted of a small 4 x 3 symbol matrix and included the same text predictive system used in the SpellermodPred. Next, we give a better description of these interfaces.

\subsubsection{Spellermod}

The Spellermod used a 7 × 6 matrix (See Figure 1). The last row contained only two special characters, BORRAR and ESPACIO, for deleting a character and introducing a blank space, respectively. Therefore, in case of a spelling error, the user was able to erase 
the last letter by selecting the special character BORRAR. Besides, the digit zero was also included in the sixth row.

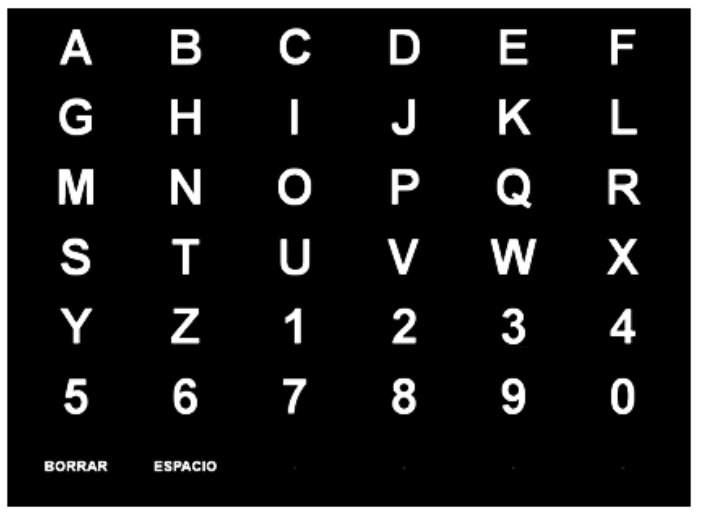

Figure 1: Interface of the Spellermod.

The characters that the user had selected were displayed inside a text box situated at the bottom of the screen. According to the temporary parameters described in section 2.1, the time needed to select a symbol through this interface was $34.5 \mathrm{~s}$.

\subsubsection{SpellermodPred}

Although the main objective of this study was to compare the SpellerT9 with the reference speller (i.e., the Spellermod), we considered very interesting to assess the performance of a classical speller when it included a text predictive system. So, we developed the SpellermodPred speller (See Figure 2). Its interface was like the Spellermod's 7 x 6 matrix, only now it included the special character VALIDAR. Since the matrix dimensions were the same as in the Spellermod, the time needed to select a symbol using the SpellermodPred was also $34.5 \mathrm{~s}$.

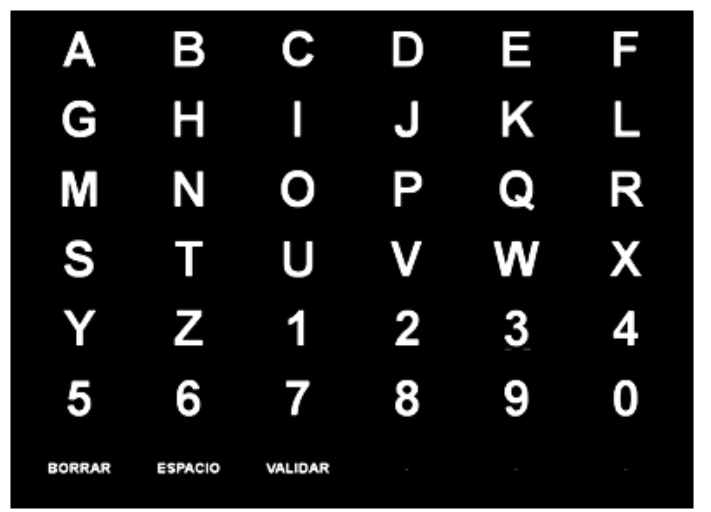

Figure 2: Interface of the SpellermodPred.

The characters (i.e., letters) that the user selected were displayed in a text box below the symbol matrix. These characters were fed into the text predic- tor, which in turn displayed the most frequently used Spanish word starting with those letters in an additional text box, just above the former. For example, if the subject had selected the letter $\mathrm{G}$ and then the letter $\mathrm{O}$, the predictor would suggest the word gobierno (i.e., the Spanish word for government).

In case the suggested word were the one the user wanted to write, he/she could confirm it by selecting the special character VALIDAR. As a consequence, the rest of the word was added to the letters he/she had already spelled, allowing the user to write a new word. Importantly, validating a word also added a blank space at the end of it.

The list of the most frequently used Spanish words was obtained from the Royal Spanish Academy. In case of wanting to write in a different language, it would only be necessary to change the dictionary used by the text predictor.

\subsubsection{SpellerT9}

The SpellerT9 used the T9 predictive text system developed for mobile phones. Its interface (See Figure 3) consisted of a $4 \times 3$ matrix of elements or keys, in which only eight keys-the ones corresponding to the numbers 2 to 9 -were used for spelling. Each of those keys corresponded to three to four letters. Importantly, as the matrix size was $4 \times 3$, the time needed to select a symbol in the SpellerT9 was just $19.5 \mathrm{~s}$, less than that of the other spellers.

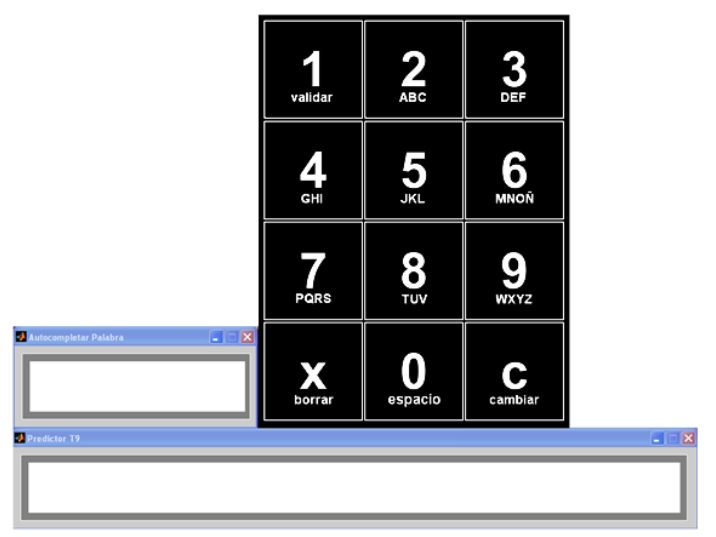

Figure 3: Interface of the SpellerT9.

As in the T9 interface of mobile phones, the user of the SpellerT9 had to select a sequence of the mentioned eight keys in order to write a word. As the user selected a sequence of those keys, the most favorable sequence of letters corresponding to those keys was displayed in the text box at the bottom of the interface. To increase writing speed, the SpellerT9 used a text predictive system, exactly like the one used in the SpellermodPred. This system identified the four 
more frequently used Spanish words starting with the suggested sequence of letters. The most frequent of them was displayed in the text box beside the keyboard and the other three were kept in memory. For example, after selecting the sequence of keys 2272 , the four predicted words would be casa, cara, capa, and basa, being casa - the most frequently used - the one displayed beside the keyboard. In case that were the word that the user wanted to write, he/she could confirm it by selecting the key 1 (i.e., validar). This, as in the SpellermodPred, added a blank space at the end of the word. Otherwise, the user could select the key c (i.e., cambiar) to switch to a new interface that displayed the four predicted words (See Figure 4). The user could then select one of them or alternatively go back to the virtual keyboard by selecting the left arrow key (i.e., volver).

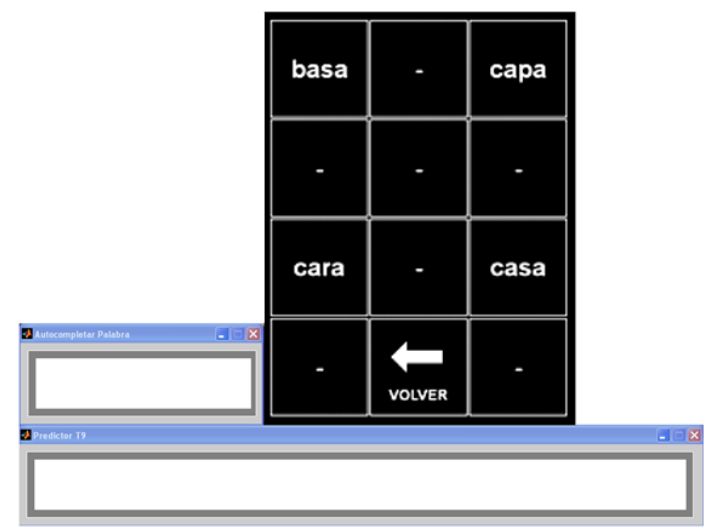

Figure 4: 4 x 3 matrix of the SpellerT9 showing the four most frequently used Spanish words associated with the sequence of keys 2272 .

Like in the other two spellers, users could delete characters - by selecting the key x (i.e., borrar) - or include blank spaces-by selecting the key 0 (i.e., espacio)-.

The SpellerT9 made it also possible for users to write digits. To do so, he/she had to select the key c prior to selecting any letter of a new word. In this way, the user could select the keys 0 to 9 to write the corresponding digits. Once the desired digits had been written, the user had to select the key c again to continue writing words.

\section{RESULTS AND DISCUSSION}

The spelling times needed by each of the three participants to write the target sentence (i.e., "Experiencia BCI en la Universidad de Málaga") through each of the three compared spellers are shown in Table 1. We did not discount the times associated with errors. The table also displays the minimum required times that a perfect user (i.e., a user that selected each character at the first attempt) would need to write the target sentence in each case.

As we can see, the three participants wrote nearly all words faster with the SpellerT9 than with any of the other two spellers. Even participant 2, who did not gain too much control of any of the three interfaces, achieved the shortest spelling time for the full target sentence when using the SpellerT9. As for the other two participants, they made very few mistakes, as their spelling times were slightly longer than the minimum required, regardless of the speller they used.

The average spelling time for the full target sentence was $650.5 \mathrm{~s}, 1115.5 \mathrm{~s}$ and $1849.5 \mathrm{~s}$ when using the SpellerT9, the SpellermodPred and the Spellermod, respectively. The average spelling times for each word when using each of the three interfaces are shown in Figure 5. These results strongly suggest that SpellerT9 system was the fastest writing interface of all three.

Furthermore, our study supports the usefulness of a text predictive system irrespective of matrix size, as participants achieved shorter spelling times when using the SpellermodPred than when using to conventional interface of the Spellermod.

\section{CONCLUSION}

The objective of this study was to propose and to validate a new P300-based BCI speller system aimed at increasing the user's writing speed. The proposed speller (i.e., SpellerT9) was based on the T9 interface developed for mobile phones. It presented a 4 x 3 virtual keyboard and incorporated the $\mathrm{T} 9$ text predictive system.

Although the study has been carried out with a very small sample, our preliminary results suggest that the SpellerT9 leads to lower spelling times than those of an adaptation of the classical Farwell and Donchin speller (i.e., Spellermod), even when the latter interface is improved by adding a text predictor (i.e., SpellermodPred). The three participants in our experiment achieved the lowest spelling times when they wrote a target sequence through the SpellerT9 and the highest when they used the Spellermod. Besides, the writing times of each subject indicate that the SpellerT9 is not more difficult to use than any of the other two spellers, as participants performed just slightly worse than a perfect user.

Nevertheless, these conclusions should be supported by extending this experiment to a greater sam- 
Table 1: Spelling times needed by the three participants to write each word of the target sentence as a function of the speller they used.

\begin{tabular}{|c|c|c|c|c|c|c|c|c|}
\hline \multirow[b]{2}{*}{ Speller } & \multicolumn{7}{|c|}{ Time (s) for each word } & \multirow[b]{2}{*}{ Total time (s) } \\
\hline & "Experiencia" & "BCI" & "en" & "la" & "Universidad" & "de" & "Málaga" & \\
\hline & \multicolumn{8}{|c|}{ Participant 1} \\
\hline Spellermod & 414 & 138 & 103.5 & 103.5 & 621 & 103.5 & 276 & 1759.5 \\
\hline SpellermodPred & 172.5 & 138 & 103.5 & 69 & 207 & 103.5 & 207 & 1000.5 \\
\hline \multirow[t]{2}{*}{ SpellerT9 } & 78 & 78 & 58.5 & 39 & 97.5 & 39 & 117 & 507 \\
\hline & \multicolumn{8}{|c|}{ Participant 2} \\
\hline Spellermod & 483 & 345 & 310.5 & 172.5 & 483 & 103.5 & 276 & 2173.5 \\
\hline SpellermodPred & 138 & 103.5 & 103.5 & 517.5 & 172.5 & 69 & 345 & 1449 \\
\hline \multirow[t]{2}{*}{ SpellerT9 } & 78 & 156 & 58.5 & 78 & 214.5 & 78 & 177 & 840 \\
\hline & \multicolumn{8}{|c|}{ Participant 3} \\
\hline Spellermod & 414 & 138 & 103.5 & 103.5 & 414 & 172.5 & 270 & 1615.5 \\
\hline SpellermodPred & 138 & 138 & 103.5 & 69 & 172.5 & 69 & 207 & 897 \\
\hline \multirow[t]{2}{*}{ SpellerT9 } & 78 & 117 & 78 & 39 & 97.5 & 39 & 156 & 604.5 \\
\hline & \multicolumn{8}{|c|}{ A perfect user } \\
\hline Spellermod & 414 & 138 & 103.5 & 103.5 & 414 & 103.5 & 270 & 1546.5 \\
\hline SpellermodPred & 138 & 103.5 & 103.5 & 69 & 172.5 & 69 & 207 & 862.5 \\
\hline SpellerT9 & 78 & 78 & 58.5 & 39 & 97.5 & 39 & 117 & 507 \\
\hline
\end{tabular}

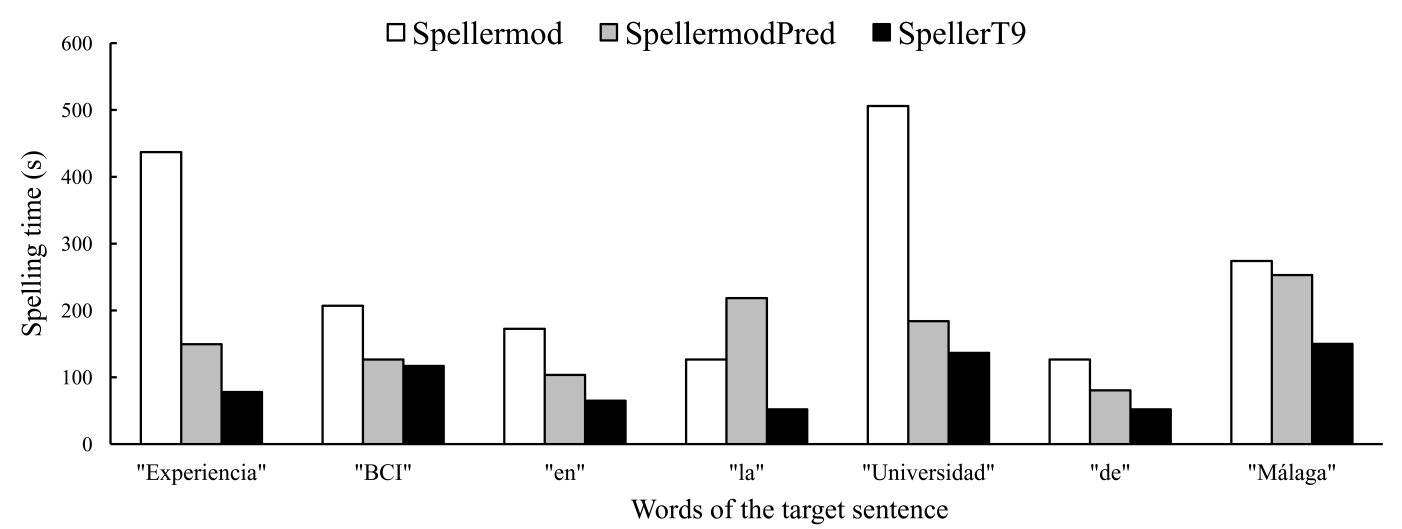

Figure 5: Average spelling times needed to write each word of the target sentence using each of the three spellers. 
ple of participants and by obtaining additional direct measures of user performance.

\section{ACKNOWLEDGEMENTS}

This work was partially supported by the Innovation, Science and Enterprise Council of the Junta de Andalucía (Spain), project P07-TIC-03310, the Spanish Ministry of Science and Innovation, project TEC 2011-26395 and by the European fund ERDF.

\section{REFERENCES}

Bianchi, L., Sami, S., Hillebrand, A., Fawcett, I., Quitadamo, L., and Seri, S. (2010). Which physiological components are more suitable for visual ERP based brain-computer interface? A preliminary MEG/EEG study. Brain Topography, 23(2):180-185.

Birbaumer, N. (2006). Breaking the silence: Braincomputer interfaces (BCI) for communication and motor control. Psychophysiology, 43(6):517-532.

Donchin, E., Spencer, K., and Wijesinghe, R. (2000). The mental prosthesis: Assessing the speed of a P300-based brain-computer interface. IEEE Transactions on Rehabilitation Engineering, 8(2):174-179.

Dunlop, M. D. and Crossan, A. (2000). Predictive text entry methods for mobile phones. Personal Technologies, 4(23):134-143.

Kececi, H., Degirmenci, Y., and Atakay, S. (2006). Habituation and dishabituation of P300. Cognitive and Behavioral Neurology, 19(3):130-134.

Kleih, S., Nijboer, F., Halder, S., and Kübler, A. (2010). Motivation modulates the P300 amplitude during braincomputer interface use. Clinical Neurophysiology, 121(7): 1023 - 1031

Kleih, S. C., Kaufmann, T., Hammer, E., Pisotta, I., Pichiorri, F., Riccio, A., Mattia, D., and Kübler, A. (in press). Motivation and SMR-BCI: Fear of failure affects BCI performance. In Proceedings of the Fifth International BCI Meeting.

Krusienski, D., Sellers, E., McFarland, D., Vaughan, T., and Wolpaw, J. (2008). Toward enhanced P300 speller performance. Journal of Neuroscience Methods, 167(1):15 -21 .

Lu, J., Speier, W., Hu, X., and Pouratian, N. (2013). The effects of stimulus timing features on $\mathrm{P} 300$ speller performance. Clinical Neurophysiology, 124(2):306 - 314.

Mak, J. and Wolpaw, J. (2009). Clinical applications of brain-computer interfaces: Current state and future prospects. IEEE Reviews in Biomedical Engineering, 2:187-199.
Mangun, G. R. and Buck, L. A. (1998). Sustained visualspatial attention produces costs and benefits in response time and evoked neural activity. Neuropsychologia, 36(3): $189-200$.

McFarland, D. J., Sarnacki, W. A., Townsend, G., Vaughan, T., and Wolpaw, J. R. (2011). The P300-based braincomputer interface (BCI): Effects of stimulus rate. Clinical Neurophysiology, 122(4):731 - 737.

Murata, A. and Uetake, A. (2001). Evaluation of mental fatigue in human-computer interaction - Analysis using feature parameters extracted from event-related potential. In 10th IEEE International Workshop on Robot and Human Interactive Communication, pages 630-635.

Polich, J. and Kok, A. (1995). Cognitive and biological determinants of P300: An integrative review. Biological Psychology, 41(2):103 - 146.

Schalk, G., McFarland, D., Hinterberger, T., Birbaumer, N., and Wolpaw, J. (2004). Bci2000: A general-purpose brain-computer interface (BCI) system. IEEE Transactions on Biomedical Engineering, 51(6):1034-1043.

Sellers, E. W. and Donchin, E. (2006). A P300-based brain-computer interface: Initial tests by ALS patients. Clinical Neurophysiology, 117(3):538 - 548.

Sellers, E. W., Krusienski, D. J., McFarland, D. J., Vaughan, T. M., and Wolpaw, J. R. (2006). A P300 event-related potential brain-computer interface (BCI): The effects of matrix size and inter stimulus interval on performance. Biological Psychology, 73(3):242 - 252.

Shih, J. J., Townsend, G., Krusienski, D. J., Shih, K. D., Shih, R. M., Heggeli, K., Paris, T., and Meschia, J. F. (in press). Comparison of checkerboard P300 speller vs. row-column speller in normal elderly and aphasic stroke population. In Proceedings of the Fifth International BCI Meeting.

Wang, C., Guan, C., and Zhang, H. (2005). P300 braincomputer interface design for communication and control applications. In 27th Annual International Conference of the Engineering in Medicine and Biology Society IEEE-EMBS 2005, pages 5400-5403.

Wolpaw, J. R., Birbaumer, N., McFarland, D. J., Pfurtscheller, G., and Vaughan, T. M. (2002). Braincomputer interfaces for communication and control. Clinical Neurophysiology, 113(6):767-791. 